\title{
Medición del clima organizacional en una Pyme del sector servicios de la región centro de Hidalgo, México.
}

\section{Measurement of the organizational climate in an SME in the service sector of the central region of Hidalgo, Mexico.}

Jocelyn Estrella Rodríguez Martínez; Enrique Martínez Muñoz;

Danae Duana Àvila3; Tirso Javier Hernández Gracia ${ }^{4}$

\section{Palabras claves: \\ Clima Organizacional, Pymes, Sector Servicios, Competitividad, Satisfacción Laboral, Gestión organizacional y Negocios, Gestión humana}

Artículo de investigación:

Fecha de recepción: 2019/06/14

Fecha de aceptación: 2019/12/16

Esta publicación se encuentra bajo licencia:

Creative Commons ReconocimientoNoComercialSinObraDerivada 4.0 Internacional

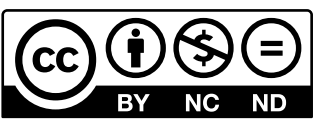

\section{Resumen}

En los últimos años, se han incrementado las Pymes, principalmente las del sector servicios, las cuales ofrecen innumerables oportunidades para estimular el crecimiento y el empleo en un país. Sin embargo, pese a que las Pymes son el pilar fundamental de las organizaciones modernas, en los últimos años se ha frenado su crecimiento debido a la baja productividad que han tenido.

Una de las debilidades de los directivos de las Pymes, es el no considerar a sus empleados como clientes internos, olvidan que ellos reflejan la filosofía, los valores, la calidad y el servicio que brinda la empresa; por lo tanto, dejan de lado el ambiente laboral y los empresarios dan por hecho que les proporcionan a sus empleados las herramientas necesarias para que desarrollen sus funciones y se desempeñen adecuadamente.

Maestrante Maestría en Ingeniería Industrial, Universidad Autónoma del Estado de Hidalgo, México jocelyn_rodriguez10259@uaeh.edu.mx, https:// orcid.org/0000-0002-0726-0740

2 Doctor en Ciencias en Administrativas, Universidad Autónoma del Estado de Hidalgo, México, emmunoz@uaeh.edu.mx, https://orcid.org/0000-00016418-5292

3 Doctor en Economìa, Universidad Autónoma del Estado de Hidalgo, México, duananos@yahoo.com.mx, https://orcid.org/0000-0003-2286-2843

4 Doctor en Ciencias en Administrativas, Universidad Autónoma del Estado de Hidalgo, México, explorerall@hotmail.com, https://orcid.org/0000-00030425-0800 


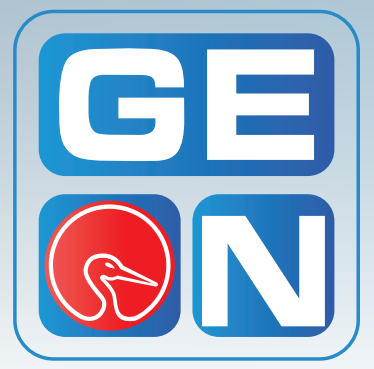

Revista GEON

(Gestión, Organizaciones y Negocios)

ISSN: 2346-3910 en línea

revistageon@unillanos.edu.co

Universidad de los Llanos

Colombia

Rodríguez Martínez, J., Martínez Muñoz, E., Duana Avila, D., \& Hernández Gracia, T. (2020).

Medición del clima organizacional en una Pyme del sector servicios de la región centro de Hidalgo.

Revista GEON

(Gestión, Organizacionesy Negocios), 7(1). 205 - 217

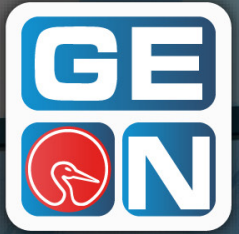

66 Una de las debilidades de los directivos de las Pymes, es el no considerar a sus empleados como clientes internos, olvidan que ellos reflejan la filosofía, los valores, la calidad y el servicio que brinda la empresagو

Rodríguez Martínez, J., Martínez Muñoz, E., Duana Avila,

D., \& Hernández Gracia, T. (2020). Medición del clima organizacional en una Pyme del sector servicios de la región centro de Hidalgo. Revista GEON (Gestión, Organizaciones Y Negocios), 7(1) $205-217$.

http://revistageon.unillanos.edu.co 
En la presente investigación, se plantea el estudio para medir el clima organizacional en una Pyme del sector de servicios del estado de Hidalgo, mediante la aplicación de un cuestionario a sus trabajadores, con la finalidad de conocer la percepción que estos tienen acerca del ambiente que les rodea en la empresa. Una vez procesada la información, se obtuvieron resultados alarmantes, los cuales indicaron que la empresa tiene un clima organizacional con un nivel bajo, esto quiere decir que los empleados carecen de motivación, compromiso y no están satisfechos.

Ante este análisis, sus dirigentes podrían implementar acciones para conducir la gestión de cambios en su clima laboral y logren planificar técnicas que influyan en los trabajadores para que en estos exista un sentimiento de pertenencia que los motive a mejorar su desempeño y con ello, se incremente la productividad, se optimicen los servicios para ser más competitivos y se aseguren la permanencia de la empresa en el mercado.

3041/PC/RR, 1702/PC/TR, 1450/PC/TE, 1858/PC/TR, 3937/PC/TR

Codigos JEL: 181

\section{Abstract}

In recent years, SMEs have increased, mainly those in the service sector, which offer innumerable opportunities to stimulate growth and employment in a country. However, despite the fact that SMEs are the fundamental pillar of modern organizations, their growth has slowed in recent years due to the low productivity they have had.

One of the weaknesses of SME managers is not considering their employees as internal customers, they forget that they reflect the company's philosophy, values, quality and service; therefore, they neglect the work environment and employers assume that they provide their employees with the necessary tools to carry out their duties and perform properly. 
Cómo citar este artículo /

To reference this article:

Rodríguez Martínez, J., Martínez Muñoz, E., Duana Avila, D., \& Hernández Gracia, T. (2020). Medición del clima organizacional en una Pyme del sector servicios de la región centro de Hidalgo. Revista GEON (Gestión, Organizaciones Y Negocios), 7(1) 205 -217. https:// doi.org/10.22579/23463910.154
In the present investigation, the study to measure the organizational climate in an SME in the service sector of the state of Hidalgo is proposed, through the application of a questionnaire to its workers, in order to know the perception that they have about the environment that surrounds them in the company. Once the information was processed, alarming results were obtained, which indicated that the company has an organizational climate with a low level, this means that the employees lack motivation, commitment and are not satisfied.

Given this analysis, their leaders could implement actions to drive the management of changes in their work environment and manage to plan techniques that influence workers so that there is a feeling of belonging that motivates them to improve their performance and thus increase productivity, services are optimized to be more competitive and the permanence of the company in the market is ensured.

Keywords: Organizational Climate, SMEs, Services Sector, Competitiveness, Job Satisfaction, Organizational Management and Business, Human Management

\section{Introducción}

En la actualidad las empresas, principalmente la del sector servicios, dependen de la atención que se le da al cliente; las compañías están conscientes que el costo de mantener a un cliente es inferior al costo de conseguir uno nuevo y a su vez ligeramente menor al de recuperar a un cliente perdido. Es por ello que se requiere tener personal altamente capacitado, que tengan una actitud positiva y alto grado de responsabilidad.

Es fundamental señalar que el clima laboral comprende la interacción en- tre factores personales cómo son: las actitudes, motivaciones, expectativas, etc., que tienen los empleados hacia la empresa y de las organizaciones como políticas, satisfacción, productividad, entre otras.

Cada factor va a determinar el comportamiento de los empleados, y por lo tanto van a incidir en el sentido de pertenencia, la resolución de problemas, la calidad de los servicios, en la atención al cliente, mismos que son la clave para el desempeño de una empresa.

Una empresa, por muy automatizada que esta sea, por sí sola no es capaz de 
producir, necesita de las actitudes, habilidades y esfuerzos de las personas, mismas que a través de la integración, cooperación y trabajo en equipo debidamente coordinado por un dirigente, podrán lograr metas y objetivos organizacionales para ser competitivas.

Las Pymes son las empresas que generan más empleos en nuestro país y aportan más a la economía; sin embargo, son las que menos posibilidades tienen de permanecer en el mercado. La principal razón es por la falta de experiencia en el ramo de los negocios por parte de los nuevos emprendedores, ya que estos generan una empresa como parte de una aspiración personal o profesional y/o mejorar sus ingresos para tener una mejor calidad de vida, dejando a un lado la parte organizativa de la empresa.

Con esta investigación se pretende determinar a través de un análisis del clima laboral en una Pyme, si el medio ambiente en las actividades que se desarrollan cotidianamente en el trabajo, influye en la productividad y por lo tanto es un determinante para la permanencia de una empresa.

La importancia de realizar un análisis de clima en una organización, radica en vislumbrar los factores que influyen en el comportamiento de los empleados, a fin de estimularlos para que se comprometan con la empresa, se sientan más cómodos, satisfechos y motivados, para que brinden al cliente una atención y servicio de calidad, y con ello contribuyan a alcanzar los objetivos planteados por la organización.

\section{Contexto teórico}

Diversos investigadores del ámbito de la Administración han remarcado que es importante realizar esfuerzos para asegurar un ambiente donde exista una sana convivencia y una adecuada coexistencia entre las personas que laboran en un lugar, con ello los trabajadores se sentirán valorados, integrados e implicados con los objetivos de la organización y es posible que tengan un desempeño más alto.

Las organizaciones según Daft (2011) "son entidades sociales que están dirigidas a las metas, están diseñadas como sistemas de actividades estructuradas y coordinadas en forma deliberada y están vinculadas al entorno"; en la opinión de Chiavenato (2007) "una organización constituye el conjunto de personas que interactúan entre si para alcanzar objetivos específicos". Ambos coinciden en que, en una organización las personas se relacionan entre sí para realizar funciones específicas que ayudaran a alcanzar las metas propuestas; por lo que es necesario contar con un clima propicio que ayude a que los empleados se sientan satisfechos y desarrollen sus funciones con eficacia.

El término clima tiene su origen en la meteorología y es visto como un fenómeno complejo, puesto que sus características se pueden medir de forma individual cómo la temperatura, las precipitaciones, los vientos, las variaciones estacionales, las horas de luz solar, entre otras.

Al trasladar al clima en las organizaciones, se habla de una serie de rasgos 
medibles que pueden variar motivados por diversas razones que influirán directamente en los individuos y, por ende, en las organizaciones.

Este concepto ha sido de interés para los estudiosos y se ha nombrado de diversas formas, cómo: atmósfera, ambiente, estado de ánimo de la organización, clima laboral, clima psicológico, entre otros; por lo que, se ha discutido en repetidas ocasiones y es motivo de múltiples investigaciones.

Brunet (2011), definió al clima organizacional como "un componente multidimensional de elementos al igual que lo es el clima atmosférico"; también indicó que son "elementos individuales relacionados con los valores, necesidades e incluso el grado de satisfacción del empleado", en otras palabras, el individuo percibe el clima organizacional en función de las necesidades que la organización le puede satisfacer.

Los estudios de clima organizacional se convierten en una poderosa herramienta para evaluar y medir factores que afecten de manera positiva o negativa, la percepción que tiene un empleado en su área de trabajo. Sus resultados permitirán realizar cambios en el entorno organizacional para mejorar los sistemas de comunicación y procesos de productos y/o servicios, resolver conflictos, reducir los niveles de rotación, entre otros.

Se han realizado recientes investigaciones del clima laboral en instituciones tanto públicas como privadas a nivel nacional e internacional, para demostrar la importancia que tiene el que un directivo conozca y mejore el ambiente en su organización.
Carlos Pardo (2018), hace alusión a la frase del cirujano francés Victor Pauchet "El trabajo más productivo, es el que sale de las manos de un hombre feliz"; esto quiere decir que las personas al sentirse bien en sus áreas de trabajo, se esforzaran más y se desempeñarán adecuadamente, lo que traerá como consecuencia que se incremente la productividad, beneficiando a la empresa.

Los autores Martínez E., Hernández T. y Duana D. (2018), realizaron un estudio del clima organizacional, la comunicación y el Liderazgo en Pymes de la industria Textil en el estado de Hidalgo, ellos mencionan que los trabajadores describieron un clima laboral bajo, en contraste con los jefes que decían tener un clima laboral bueno, esto se debió a que las percepciones que tienen los trabajadores poseen un significado motivacional, personal o emocional, por otra parte, los jefes, expresan sus percepciones considerando aspectos precisos de la empresa que tiene incidencia en el compromiso organizacional. Este estudio da pauta para impulsar el cambio en estas pequeñas empresas industriales y con ello mejorar su ambiente laboral.

Los investigadores Erazo, W., Claudio, B., \& Erazo, J. (2018), realizaron un estudio en empresas del sector servicios acantonadas en Rumiñahu, Ecuador y se evidenció que el $14.3 \%$ de las empresas analizadas presentan un clima organizacional aceptable, esto se debe principalmente a la falta de planificación estratégica en las empresas, lo cual es un factor clave en el crecimiento de las organizaciones. 
Los autores recomiendan diseñar planes o programas orientados a un cambio organizacional que promueva el mejoramiento y fortalecimiento del clima laboral en las empresas.

Por otro lado, Gómez, L. A., Balderas, H. C., \& Rangel, D. O. (2016), realizaron un diagnóstico del clima laboral en una empresa familiar conocida como Distribuidora Rachel ubicada en el estado de Coahuila, México; determinaron que clima organizacional de la empresa es clave para el éxito por que condiciona las actitudes y el comportamiento de sus trabajadores y concluyeron que las condiciones del ambiente de trabajo y actitudes de los trabajadores si están determinadas por el clima laboral de la empresa.

Los autores Méndez F., Natalia M., Echeverri C., Rico J., Ricardo L. (2016), mencionan que el realizar en una Pyme un análisis del clima organizacional determinará factores vinculados con la calidad de vida de los trabajadores, permitirá considerar claves en el proceso elementales, como el liderazgo, comunicación, toma de decisiones, relaciones interpersonales, el ambiente, tecnología, ubicación, capacitación, salarios. El conjunto de estos desde un enfoque que favorezca al empleado, se traducirán en productividad y crecimiento económico y organizacional.

Los investigadores Jing, Avery, \& Bergsteiner (2011), realizaron un estudio sobre pequeñas farmacias de Sídney, ellos señalan que, los administradores o gerentes de estas empresas, son los que determinan los niveles de clima organizacional. Estos autores sugieren que su estudio debería ser discutido y se deberían ampliar sus hallazgos en otros sectores productivos.

En un artículo de Pymempresario (2019), se hace mención que, de acuerdo con la Condusef, el 72\% del empleo en México es generado por cerca de 4.1 millones de PyMEs en el país y según datos del Centro de Desarrollo para la Competitividad Empresarial, sólo el 75\% logra superar los dos años de vida, debido a factores externos del sector económico y a factores internos como la falta de control administrativo.

Un artículo de El Financiero (2018), hace alusión que las Pymes aportan el $42 \%$ del Producto Interno Bruto, sin embargo, debido a obstáculos externos y a errores internos que los empresarios cometen al dirigir sus empresas, hacen que frenen su crecimiento y, por lo tanto, este sector no se fortalezca de manera adecuada; se hace hincapié en que se debe invertir en la atracción y retención del talento adecuado, ya que es la clave para lograr las metas del negocio.

Erazo et al. (2018), mencionan que, en Ecuador, las Pymes representan el 4,3\% del total de empresas que generan empleo en un $24 \%$ y poseen el $15 \%$ del total las de ventas generadas a nivel interno. Estos datos lo ubican por encima del 50\% de los países de Latinoamérica.

En este contexto es importante estudiar el impacto que tiene el clima organizacional en el desempeño de los trabajadores de las pequeñas y medianas empresas, que en este caso, la presente investigación se enfocará en una empresa de multiservicios, ubicada en estado de Hidalgo, Méxi- 
co, para lo cual que se utilizó el instrumento desarrollado por el Dr. Enrique Martínez Muñoz, quién ha desarrollo diversas investigaciones en le ámbito de las organizaciones y cuya confiabilidad del instrumento tiene un Alpha de Cronbach del 83\%.

\section{Materiales y métodos}

El instrumento de medida utilizado por excelencia en los estudios de evaluación del clima organizacional, es el cuestionario escrito. Este tipo de instrumento contiene preguntas que se adecuan a las necesidades de las empresas y que describen hechos particulares de la organización, sobre los cuales deben indicar hasta qué punto están de acuerdo o no con esta descripción.

Su medición puede proporcionar herramientas a los directivos que permitan modificar sus conductas para crear un buen clima organizacional y consigan orientar sus esfuerzos hacia los aspectos que logre mayores niveles de eficiencia y cumplimiento de metas por la acción de los empleados.

Por lo anterior, se aplicó un cuestionario a los trabajadores que laboran en la empresa Atrovisa S.A. de C.V., esta es una empresa de multiservicios que se ubica en la región centro del estado de Hidalgo, México. Se obtuvo una muestra representativa de 100 individuos y los trabajadores seleccionados, fueron contactados en su lugar de trabajo e invitados a responder el cuestionario. Para procesar la información, se utilizó el paquete estadístico SPSS (STATISTICS PACKA-
GE SCIENCES SOCIAL) para Windows versión 25 en español.

El instrumento utilizado, mide la calidad del clima organizacional para las Pymes del sector se servicios. Dicho cuestionario está constituido por dos secciones: al inicio se tienen las variables control y se solicita información cómo: género, edad, nivel académico, estado civil, departamento de adscripción y años de antigüedad en la empresa, con la finalidad de conocer si éstas variables influyen en las dimensiones del clima laboral.

La segunda parte del cuestionario se compone de 42 reactivos, con una puntuación en escala de Likert de cinco puntos medida, el cual fue utilizado para recolectar datos acerca de cómo los empleados de la empresa perciben su ambiente laboral, considerando seis dimensiones que miden el clima organizacional (Imagen 1).

Imagen 1. Dimensiones del Clima Organizacional

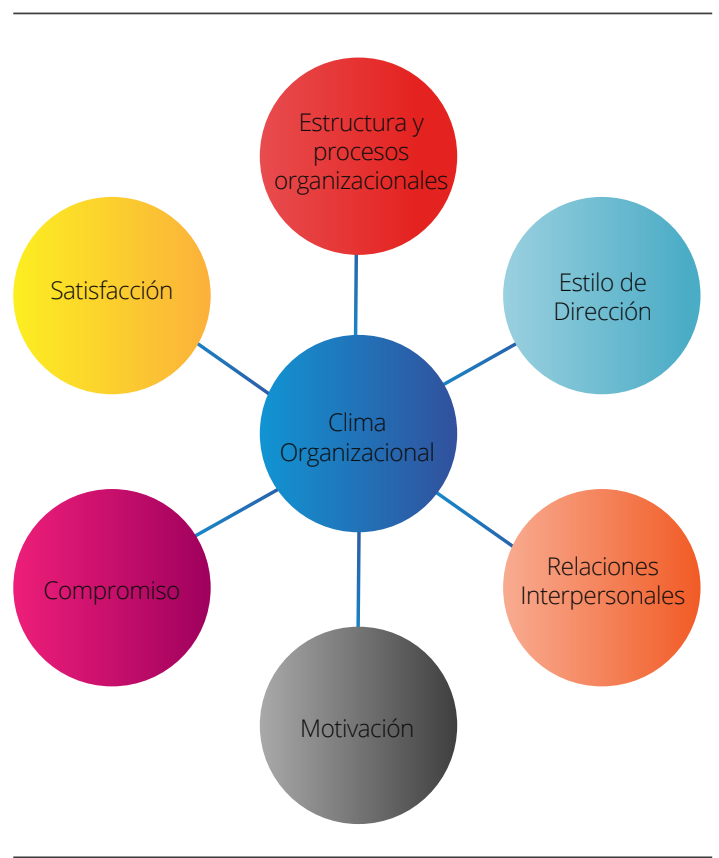

Fuente: Desarrollo propio 


\section{Resultados}

Una vez procesada la información, se obtuvieron los siguientes resultados:

Serie de gráficos 1. Género y Departamento

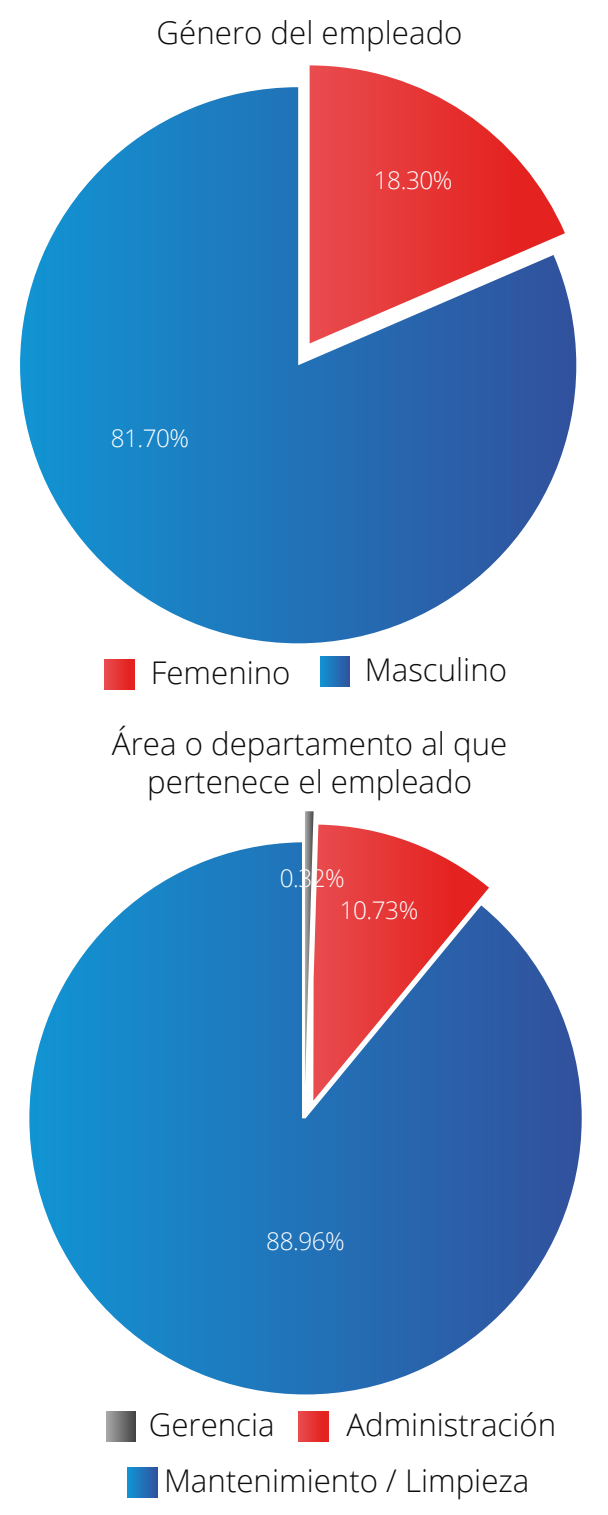

Fuente: Desarrollo propio obtenida mediente SPSS

En la serie de gráficos 1 se puede observar que la empresa de este sector, contrata más hombres debido a que no hay mucha mano de obra del sexo femenino en los oficios requeridos, cómo plomería, herrería, electricidad, entre otros; y por el tipo de giro, existen más trabajadores que están en el área de mantenimiento y limpieza.

Serie de gráficos 2. Antigëdad y Compromiso

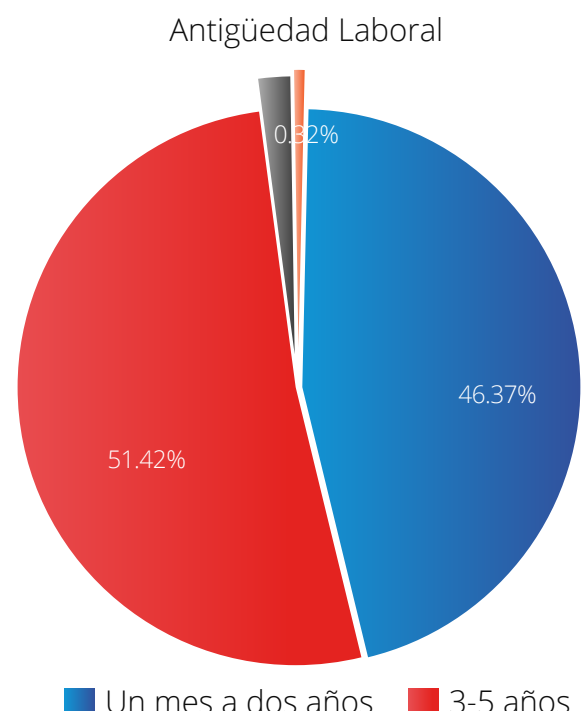

6-8 años 10 años o más Sentido de pertenencia

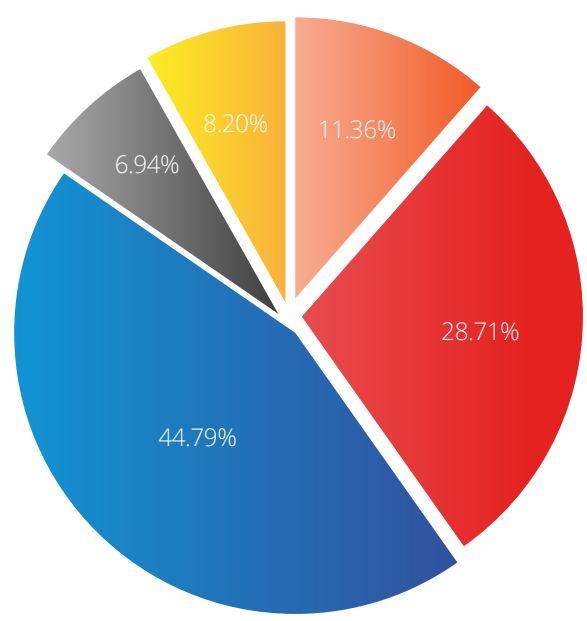

Poco Regular Suficiente

Mucho Bastante

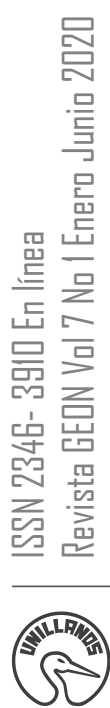


La serie de gráficos 2 muestra que los empleados tienen poco tiempo de haber ingresado a la empresa, lo cual influyó para que no se sientan totalmente comprometidos con la empresa.

Serie de gráficos 3. Opinión y Confianza

Se toma en cuenta la opinión del empleado

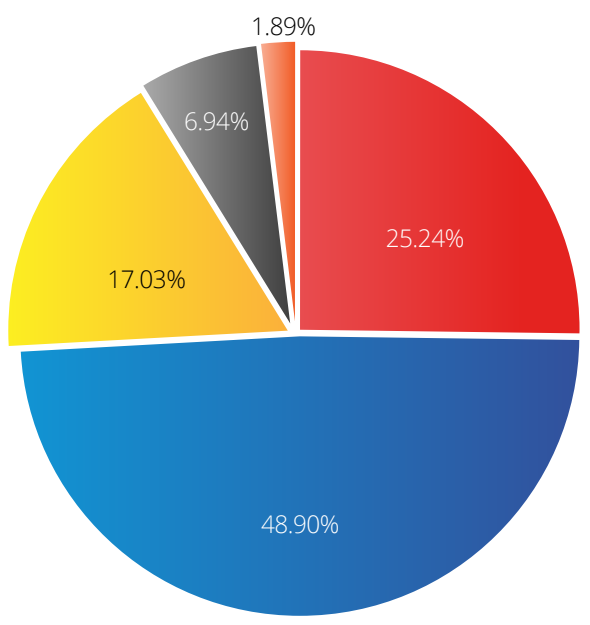

Poco Regular Suficiente

Mucho Bastante

Existe confianza en las capacidades del empleado

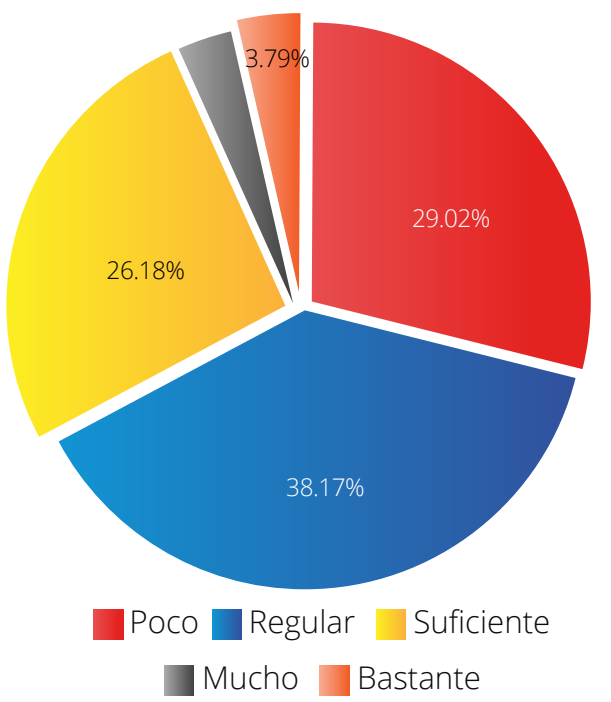

Fuente: Desarrollo propio obtenida mediente SPS
En la serie de gráficos 3 los empleados manifiestan que, prácticamente no se toman en cuenta las opciones más creativas que aportan los empleados para la solución de problemas dentro de la empresa y esto se debe a que los jefes no confían totalmente en las capacidades de los empleados.

Serie de gráficos 4. Equidad y Reconicimiento

Equidad entre sueldo y responsabilidad

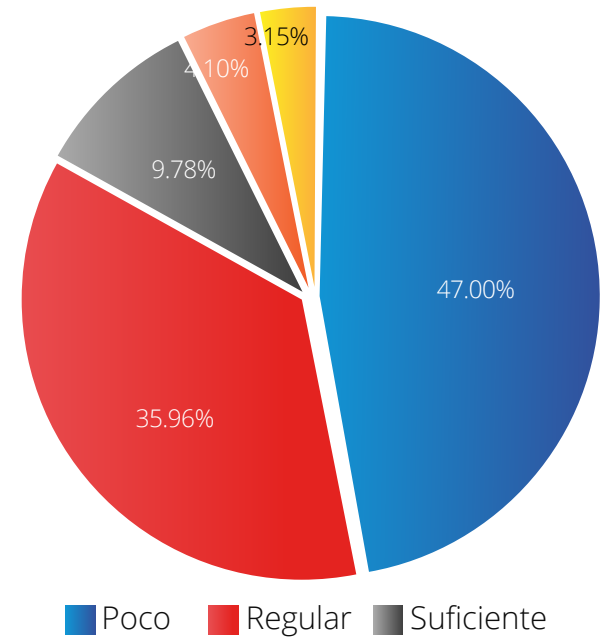

Mucho Bastante

Reconocimiento por trabajo bien realizado

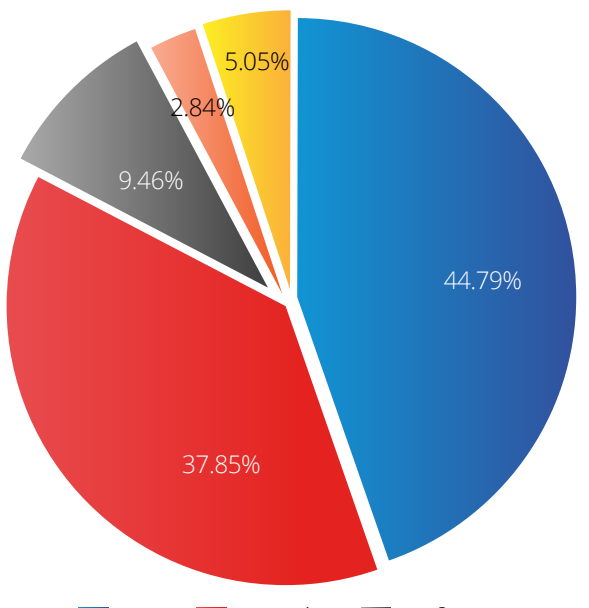

Poco Regular Suficiente

Mucho Bastante

Fuente: Desarrollo propio obtenida mediente SPS 
La serie de graficos 4 muestra que los trabajadores mencionan que existe poca equidad entre las responsabilidades que tienen y el sueldo que perciben; así mismo por lo general, los jefes no reconocen cuando el trabajador realiza un buen trabajo.

Tabla 1. Resultados de las Dimenciones

\begin{tabular}{ccc} 
Dimensiones & Concepto & $\begin{array}{c}\text { Calidad del } \\
\text { Clima }\end{array}$ \\
D1 & $\begin{array}{c}\text { Estructura y } \\
\text { procesos } \\
\text { organizacionales }\end{array}$ & 13.5 \\
D2 & BAJO \\
& Coordinación & 9.5 \\
D3 & Relaciones & BAJO \\
& Interpersonales & REGULAR \\
D4 & Motivación & 9.5 \\
& & BAJO \\
D5 & Compromiso & 11.5 \\
& & BAJO \\
D6 & Compromiso & 15.5 \\
& & BAJO \\
Calidad del clima en la empresa & BAJO \\
\hline
\end{tabular}

Fuente: Desarrollo propio

En la Tabla 1, se muestran los resultados obtenidos de las seis dimensiones que midieron el clima organizacional e indican que la empresa tiene un clima laboral bajo al alcanzar un total de 74.0 puntos, debido a que los empleados les falta más conocimiento acerca de la misión y objetivos organizacionales; los trabajadores perciben que no existe suficiente comunicación con sus jefes y que estos no confían en sus habilidades y aptitudes, no toman en cuenta sus aportaciones, carecen de un adecuado sistema de incentivos y reconocimientos por un trabajo bien realizado, por lo que los trabajadores no se identifican plenamente con la empresa.

\section{Conclusiones}

En la primera dimensión sobre la estructura y procesos organizacionales, se obtiene un resultado de 13.5 lo cual implica que dicha dimensión es baja. Esto significa que el trabajador percibe que tiene poco conocimiento acerca de los objetivos, políticas y procedimientos de la empresa, lo que conlleva a que no tenga información suficiente para atender y dar respuesta oportuna a los clientes.

En la dimensión referente a la coordinación que debe de existir entre trabajadores, el resultado fue de 9.5, que indica que es baja esto es porque que el trabajador percibe que no existe espíritu de ayuda y colaboración en la empresa y no hay apertura para expresar sus ideas.

En la tercera dimensión relaciones Interpersonales el resultado que se obtuvo fue de 14.5 lo que implica una valoración regular, porque el trabajador observa que no existe suficiente comunicación e interacción entre los miembros de la empresa.

En la dimensión respecto a la motivación, el resultado obtenido fue 9.5 lo cual implica un valor bajo porque el trabajador considera que sus condiciones laborales o salariales no son justas y equitativas.

En la quinta dimensión compromiso, el resultado obtenido fue de 11.5 lo cual nos indica un valor bajo, esto es debido a que el trabajador no tiene sentido de pertenencia ni se identifica con la organización.

El resultado de la última dimensión satisfacción, es de 15.5, lo que refle- 
ja una puntuación baja puesto que el trabajador considera que no cuenta con las herramientas necesarias para realizar sus labores, esto aunado a la falta de compensación y reconocimiento por parte de los directivos de la empresa.

La empresa al contar con clima laboral defiente, afectará la conducta del empleado, sus procesos, su toma de decisiones, la solución que le dé a los problemas que se le presenten y, por consiguiente, en su eficiencia para interactuar con el cliente, lo cual resulta perjudicial para la Pyme y pondría en riesgo su permanencia en el mercado.

Para que la empresa eleve la calidad de su clima laboral, deberá capacitar a su personal, reconocer su esfuerzo, lo remunerará y compensará adecuadamente, para que el empleado se sienta identificado y se comprometa con la organización, logrando que trabaje en equipo, este motivado y satisfecho; lo que traerá como consecuencia, que los trabajadores tengan una buena disposición a la hora de brindar un servicio y sean más capaces para dar respuesta oportuna a la problemática que enfrenten.

Con este estudio se confirma, que el clima organizacional puede ser un vínculo positivo dentro de una organización o un obstáculo en su desempeño; los empleados son un factor clave para el éxito de una empresa; su lealtad, entusiasmo, habilidades, entre otras variables contribuyen en los resultados de productividad, estos pueden hacer que una Pyme se diferencie de su competencia a través de la atención que brinda a sus clien- tes; y una vez logrado, incluso podría certificarse y será parte del Ránking de las mejores empresas con mejor clima laboral que realiza cada año la organización Great Place to Work (GPTW'TM $)$.

La presente investigación puede aportar al conocimiento de las Pymes del sector servicios, en vías de incrementar su productividad, implementando métodos para mejorar su clima laboral. De esta manera, los resultados de esta investigación pueden ser útiles para el crecimiento y competitividad de estas empresas con una mirada más clara y precisa para mejorar su la atención en el servicio al cliente, partiendo de la imagen que las organizaciones proyectan al exterior.

Se exhorta a que constantemente se evalúe esta variable, de tal manera que se puedan garantizar beneficios recíprocos (trabajador - empresa), puesto que, al existir un clima organizacional favorable, los empleados se comprometerán con el logro de objetivos y metas organizacionales, lo que traerá como consecuencia un incremento en la rentabilidad de la organización.

\section{Referencias}

Arana D. (2018). Pymes mexicanas, un panorama para 2018. Revista electrónica Forbes México. Recuperado de: https://www.forbes.com.mx/pymes-mexicanas-un-panorama-para-2018/

Brunet L. (2011). El clima del trabajo en las organizaciones, definición, diagnóstico y consecuencias. México: Trillas. 
Cantú Delgado, H. (2011). Desarrollo de una Cultura de Calidad (4ta edición ed.). México: McGrawHill Educación.

Chiavenato, I., Mascaro Sacristan, P., \& Roa, H. (2007). Administracion de recursos humanos: El capital humano de las organizaciones. McGraw-Hill Interamericana.

Chiavenato Idalberto. (2011). Administración de Recursos Humanos. México: Mc Graw Hill.

Chiavenato Idalberto. (2014). Introducción a la Teoría General de la Administración. México: Mc Graw Hill.

Cruz A. (2018). CAC: Qué es el Costo de Adquisición del Cliente y cómo calcularlo usando el Lifetime Value. RD Station. Recuperado de: https://www. rdstation.com/es/blog/costo-de-adquisicion-del-cliente/

Daft, R. L. (2011). Teoría y diseño Organizacional, décima edición, editorial Cengage Learning. Recuperado de: http:// ineurona.com/apunte/view/2484/ teora-y-diseo-organizacional-richard-daft-10ma-edicion-pdf-upc

El Financiero. (2018). 10 errores que frenan el crecimiento de las Pymes en México. Recuperado de: https://www. elfinanciero.com.mx/pyme/10-errores-que-frenan-el-crecimiento-de-laspymes-en-mexico

Erazo, W., Claudio, B., \& Erazo, J. (2018). El clima organizacional las pequeñas y medianas empresas del sector servicios. Quito, Pichincha, Ecuador.

Gómez, L. A., Balderas, H. C., \& Rangel, D. O. (2016). Diagnóstico del clima laboral en una empresa PyMes del sector servicios. Red Internacional de Investigadores en Competitividad, 9(1), 1090-1102. Recuperado de: https://riico.net/index.php/riico/article/view/61
Goncalves, A. (2000). Fundamentos del clima organizacional. Sociedad Latinoamericana para la calidad (SLC) Graw Hill.

Grueso Hinestroza, M. P. (Ed.). (2016). Organizaciones saludables y procesos organizacionales e individuales: Comprensión y retos. Editorial Universidad del Rosario.

Feng Jing, F., Avery, G.C. and Bergsteiner, H. (2011), "Organizational climate and performance in retail pharmacies", Leadership \& Organization Development Journal, Vol. 32 No. 3, pp. 224-242. https://doi. org/10.1108/01437731111123898

Martínez Muñoz, E., Hernández Gracia, T., Torres-Flórez, D., García Velázquez, M. del R., \& Montaño Arango, O. (2018). La realidad comunicacional, liderazgo y clima laboral de la pequeña empresa industrial textil en el Estado de Hidalgo. Revista GEON (Gestión, Organizaciones Y Negocios), 5(1), 76-90. https:// doi.org/10.22579/23463910.15

Mejore el clima organizacional de su pyme y aumente su eficiencia. (18 de febrero de 2016). El Financiero. Recuperado de: https://www.elfinancierocr. com/pymes/mejore-el-clima-organizacional-de-su-pyme-y-aumente-sueficiencia/ eficiencia/ MOVQLG3ZVFFMTHHQ3H4BSTEY3A/story/Pymes

Méndez F., Natalia M., Echeverri C., Rico J., Ricardo L. (2016). Importancia del Clima Organizacional para la Competitividad de las Pymes. PYMES: Gestión y Clima Organizacional. Astelio Silvera Sarmiento (Comp.). Barranquilla: Sello Editorial Corunimaricana, 95-118.

Miranda A. (2018). Pymes mexicanas: llegó el momento de apostar por la tecnología. Revista electrónica Forbes México. Recuperado de: https:// www.forbes.com.mx/brand-voice/ 
pymes-mexicanas-llego-el-momento-de-apostar-por-la-tecnologia/

Pymempresario. (10 de abril de 2019). Detectan 5 factores para el crecimiento de las PyMEs. Recuperado de: $\quad$ https://www. pymempresario. com/2019/04/detectan-5-factores-para-el-crecimiento-de-las-pymes/

Prado C. (2018). El trabajo más productivo, es el que sale de las manos de un hombre contento. Glocalthinking. Recuperado de: https://www.glocalthinking.com/el-trabajo-mas-productivo-es-el-que-sale-de-las-manos-deun-hombre-contento
Sampieri, R. H., Valencia, S. M., \& Soto, R. C. (2014). Construcción de un instrumento para medir el clima organizacional en función del modelo de los valores en competencia. Contaduría y administración, 59(1), 229257. Recuperado de: https://www. sciencedirect.com/science/article/pii/ S0186104214712501

Uribe, J. (2015). Clima y ambiente organizacional: trabajo, salud y factores psicosociales. El Manual Moderno, SA de CV ed, 31. 